\title{
Personal Exposure to PM2.5 Oxidative Potential in Association with Pulmonary
} Pathophysiologic Outcomes in Children with Asthma

\section{Linchen $\mathrm{He}^{1,2}$; Christina Norris ${ }^{3}$; Xiaoxing Cui ${ }^{1}$; Zhen $\mathrm{Li}^{4}$; Karoline K Barkjohn ${ }^{3}$; Collin}

Brehmer5; Yanbo Teng ${ }^{6}$; Lin Fang ${ }^{7,8}$; Lili Lin ${ }^{4}$; Qian Wang ${ }^{4}$; Xiaojian Zhou ${ }^{4}$; Jianguo Hong ${ }^{4}$; Feng Li ${ }^{9}$; Yinping Zhang ${ }^{7,8}$; James J Schauer ${ }^{4}$; Marilyn Black ${ }^{10}$; Michael H Bergin ${ }^{3}$; Junfeng (Jim) Zhang ${ }^{1,2,6, *}$

${ }^{1}$ Nicholas School of the Environment, Duke University, Durham, NC, USA;

${ }^{2}$ Duke Global Health Institute, Duke University, Durham, NC, USA;

${ }^{3}$ Department of Civil and Environmental Engineering, Duke University, Durham, NC, USA;

${ }^{4}$ Department of Pediatrics, Shanghai General Hospital, Shanghai Jiao Tong University, Shanghai, China;

${ }^{5}$ Department of Civil and Environmental Engineering, College of Engineering, University of Wisconsin-Madison, Madison, WI, USA;

${ }^{6}$ Duke Kunshan University, Kunshan, Jiangsu Province, China;

${ }^{7}$ Department of Building Science, Tsinghua University, Beijing, China;

${ }^{8}$ Beijing Key Laboratory of Indoor Air Quality Evaluation and Control, Beijing, China;

${ }^{9}$ Department of Pulmonary Medicine, Shanghai Chest Hospital, Shanghai Jiao Tong University, Shanghai, China;

${ }^{10}$ Underwriters Laboratories, Inc, Marietta, GA, USA.

*Corresponding author: Junfeng (Jim) Zhang, PhD, 308 Research Drive, LSRC Room A309, Durham, NC 27708, USA. Phone: (919) 681-7782. Fax: (919) 613-8061. Email: junfeng.zhang@ duke.edu

This supporting information includes:

Number of pages: 19

Number of tables: 5

29 


\section{Exposure assessment: $\mathbf{P M}_{2.5}$ compositions and $\mathbf{P M}_{2.5}$ oxidative potential}

\subsection{Personal $\mathrm{PM}_{2.5}$ mass exposure and $\mathrm{O}_{3}$ exposure}

We used an integrated sensor box, equipped with a Plantower PMS3003 sensor for $\mathrm{PM}_{2.5}$ and an Alphasense sensor (OX-A4) for $\mathrm{O}_{3}$, to simultaneously and continuously measure $\mathrm{PM}_{2.5}$ mass and $\mathrm{O}_{3}$ concentrations both in children's bedrooms and outside a window of their homes. The ambient hourly averages of $\mathrm{PM}_{2.5}, \mathrm{O}_{3}$, temperature, and relative humidity during the entire study period were obtained from the government monitoring station closest to the research clinic ( 9 $\mathrm{km}$ away). To achieve a more precise estimate, we further calculate the ambient air pollutant concentrations by averaging the pollutant concentrations measured by all the outdoor sensors and the governmental monitoring station. According to the literature data for similar building structure, various indoor/outdoor (I/O) ratios (Table S1) were used to calculate hourly average $\mathrm{PM}_{2.5}$ and $\mathrm{O}_{3}$ concentrations in different indoor microenvironments where no actual measurements were obtained.

Table S1. I/O ratio for each microenvironment.

\begin{tabular}{lcc} 
& \multicolumn{2}{c}{ I/O Ratio } \\
& $\mathbf{P M}_{2.5}$ Mass & $\mathbf{O}_{\mathbf{3}}$ \\
Bedroom (window opened) & 1 & 0.85 \\
Bedroom (window closed, during the filtration period, mean & $0.44 \pm 0.60$ & $0.35 \pm 0.42$ \\
home-specific I/O ratio \pm SD) & & \\
Bedroom (window closed, during the no filtration period) & 0.8 & 0.35 \\
Car/Taxi & 0.8 & 0.4 \\
Subway & 0.8 & 0.35 \\
Walk/ Bike/outdoor exercise & 1 & 1 \\
Other indoor environment outside home (window closed) & 0.8 & 0.35 \\
Other indoor environment outside home (window opened) & 1 & 0.85 \\
\hline
\end{tabular}

At each clinical visit, participants were asked to record their time-activity patterns for the two weeks preceding the visit on a questionnaire. Specifically, they were asked to indicate their location from the following choices for each hour: bedroom, other rooms at home, outdoors, car, subway, or other indoor environments (e.g. classroom and cafeteria). For time spent indoors, participants were asked to indicate if the window of the room was open at that time.

Personal exposures to $\mathrm{PM}_{2.5}$ mass and $\mathrm{O}_{3}$ during each hour were calculated by multiplying the location factor $(0=$ not in the location, $1=$ in the location) by the air pollutant level at each of the corresponding microenvironments during the corresponding time period and summing them up (Formula 1). Based on the hourly exposure, we calculated 24-hour average $\mathrm{PM}_{2.5}$ mass and $\mathrm{O}_{3}$ exposure zero to three days prior to each of the clinical visits. The detailed method of calculating personal $\mathrm{PM}_{2.5}$ mass and $\mathrm{O}_{3}$ exposure was published previously. ${ }^{1}$

\section{Formula 1}

$$
E X P_{i j t}=\sum_{k=1}^{6} C_{i j t k} * L_{i j t k}
$$


Code Book

$63 k$ : Microenvironment (1: bedroom, 2: other room at home, 3: car, 4: subway, 5: other indoor

64 environment outside home, 6 : outdoor)

$65 i$ : Participant id number $(i=1,2, \ldots, 43)$

$66 j$ : Visit number $(j=1,2,3,4)$

$67 t$ : Hour before health indicators measurement $(t=1,2, \ldots, 96)$

$68 E X P_{i j t}$ : Hourly personal exposure to $\mathrm{PM}_{2.5}$ mass and $\mathrm{O}_{3}$ for study participant $i$ at hour $t$ at visit $j$

$69 C_{i j t k}$ : Air pollutant concentration in microenvironment $k$ for participant $i$ at hour $t$ for visit $j$

$70 L_{i j t k}$ : Location factor for microenvironment $k$ for participant $i$ at hour $t$ for visit $j$ (0: not in the

71 microenvironment or 1: in the microenvironment)

72

73

74

75

76

77

78

79

80

81

82

83

84

85

86

87

88

89

90

91

92

93

94

95

96

97

98

99

100

101

102

\subsection{PM2.5 composition and $\mathrm{PM}_{2.5}$ oxidative potential}

Time-integrated 48-hr indoor and outdoor $\mathrm{PM}_{2.5}$ stationary samples were collected on $37 \mathrm{~mm}$ PTFE (Teflon) filters and $37 \mathrm{~mm}$ quartz filters for measuring the composition and oxidative potential of $\mathrm{PM}_{2.5}$. The indoor and outdoor $\mathrm{PM}_{2.5}$ filter samplers were placed near the integrated sensor box. Two sets of filter samples were collected at each participating household, one set during the first and one set during the second intervention session.

The concentrations of five major components of $\mathrm{PM}_{2.5}$ (organic matter, elemental carbon, sulfate, trace elements, and dust) were measured for each sample using a method previously reported. ${ }^{2}$ The concentration of "other" species not quantified was found by subtracting the sum of the five measured species from total $\mathrm{PM}_{2.5}$ mass. The percentage of these six components comprising total $\mathrm{PM}_{2.5}$ mass for each filter sample was calculated. The oxidative potential of the $\mathrm{PM}_{2.5}$ was determined using an in vitro rat alveolar macrophage cells model (NR8383 cells). The oxidative potential of $\mathrm{PM}_{2.5}$ extracts was reported as normalized to $\mu \mathrm{g} Z \mathrm{Zmosan} / \mu \mathrm{g} \mathrm{PM}_{2.5}{ }^{3}$

We assumed that the composition and oxidative potential of indoor $\mathrm{PM}_{2.5}$ samples collected during an intervention period is representative of the indoor $\mathrm{PM}_{2.5}$ for that whole 2-week filtration period. In addition, we assumed that the results of indoor $\mathrm{PM}_{2.5}$ samples collected during the period using purifiers with only the coarse filter reflect the indoor $\mathrm{PM}_{2.5}$ for the sessions with no filtration. On the other hand, all the outdoor filter data were combined and chronologically ordered. They were coupled with ambient $\mathrm{PM}_{2.5}$ mass concentration to calculate ambient $\mathrm{PM}_{2.5}$ composition and oxidative potential.

Personal exposure to specific components of $\mathrm{PM}_{2.5}$ and oxidative potential were calculated following a method similar to that used to calculate personal exposure to $\mathrm{PM}_{2.5}$ mass and $\mathrm{O}_{3}$, as follows:

\section{Formula 2}

$$
E X P_{-} C_{o m p} p_{i j t s}=\sum_{k=1}^{6} C_{i j t k} * \operatorname{Comp}_{i j t k s} * L_{i j t k}
$$


Formula 3

$$
E X P_{-} O P_{i j t}=\sum_{k=1}^{6} C_{i j t k} * O P_{i j t k} * L_{i j t k}
$$

105

\section{Code Book}

$107 k$ : Microenvironment (1: bedroom, 2: other room at home, 3: car, 4: subway, 5: other indoor 108 environment outside home, 6: outdoor)

$109 \quad i$ : Participant id number $(i=1,2, \ldots, 43)$

$110 j$ : Visit number $(j=1,2,3,4)$

$111 t$ : Hour before health indicators measurement $(t=1,2, \ldots, 96)$

112 s: $\mathrm{PM}_{2.5}$ component (1: organic matter, 2: elemental carbon, 3: sulfate, 4: trace elements, 5: dust, 113 6: other)

114 EXP_Comp E $_{i j s}$ : Hourly personal exposure to a specific component of $\mathrm{PM}_{2.5}$ for study participant $115 \quad i$ at hour $t$ for visit $j$ for specific component $s$.

116 EXP_OP $P_{i j t}$ : Hourly personal exposure to $\mathrm{PM}_{2.5}$ oxidative potential for study participant $i$ at hour $117 t$ for visit $j$ for specific component $s$.

$118 C_{i j t k}: \mathrm{PM}_{2.5}$ mass concentration in microenvironment $k$ for participant $i$ at hour $t$ for visit $j$

$119 L_{i j t k}$ : Location factor for microenvironment $k$ for participant $i$ at hour $t$ for visit $j$ (0: not in the 120 microenvironment or 1: in the microenvironment)

121 Comp $_{i j t k s}$ : Percentage of $\mathrm{PM}_{2.5}$ component $s$ in total $\mathrm{PM}_{2.5}$ mass of microenvironment $k$ for 122 participant $i$ at hour $t$ for visit $j$

$123 O P_{i j t k}$ : Oxidative potential per unit mass of $\mathrm{PM}_{2.5}$ in microenvironment $k$ for participant $i$ at hour $124 t$ for visit $j$ 
Figure $\mathbf{S 1}$ is a schematic showing the entire approach to determining the personal exposures to $\mathrm{PM}_{2.5}$ mass, composition, and oxidative potential.

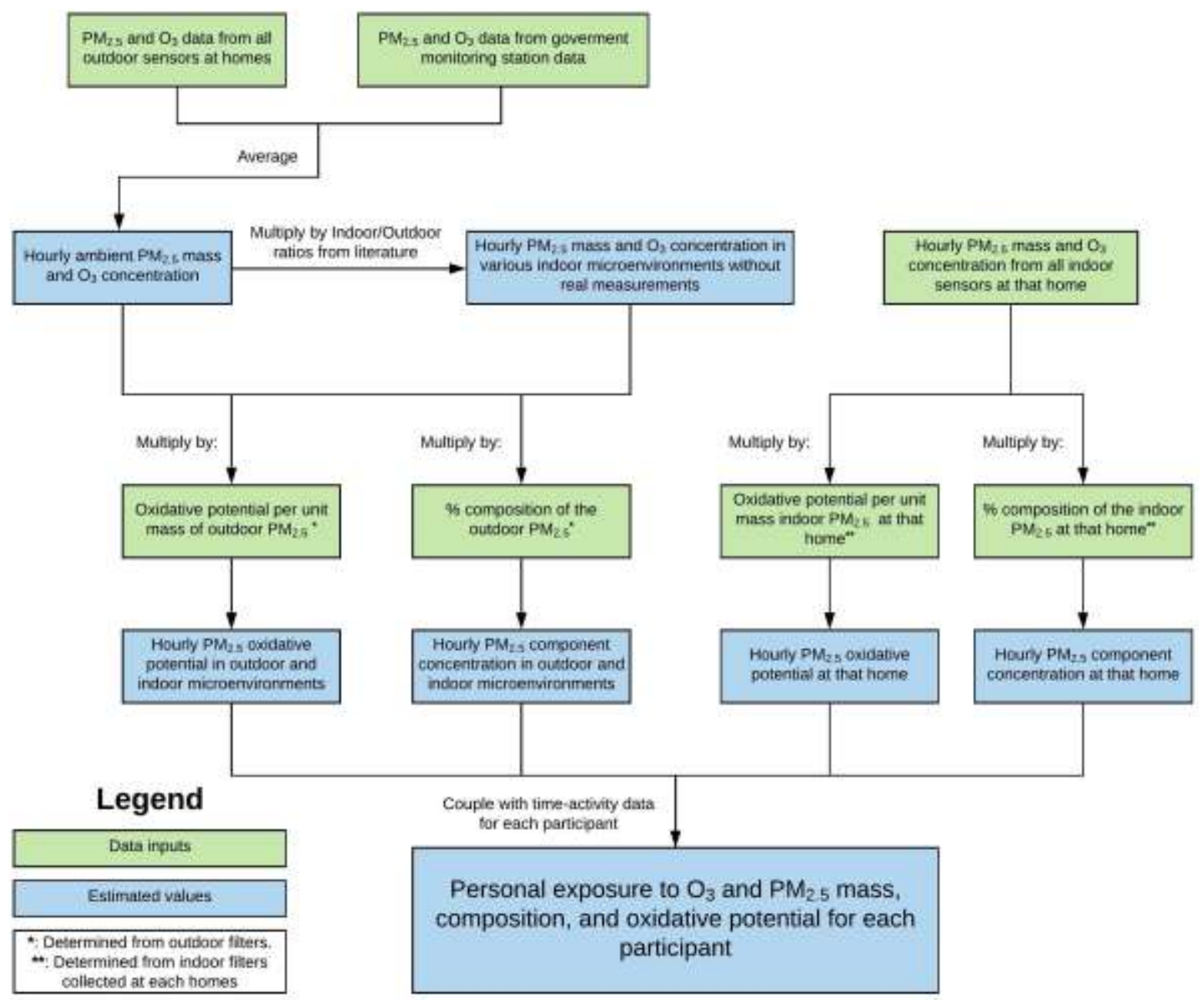

Figure S1. Schematic depicting the entire approach to calculate personal pollutant exposures. 
132 Table S2. The questions in the C-ACT questionnaire. ${ }^{4}$

Questions answered by child (score range: 0-3; 0: Very bad, 1: Bad, 2: Good, 3: Very good)

\begin{tabular}{|l|l|l|}
\hline 1 & Asthma control & How is your asthma today? \\
\hline 2 & $\begin{array}{l}\text { Limitation of physical } \\
\text { activities }\end{array}$ & $\begin{array}{l}\text { How much of a problem is your asthma when you run, exercise or } \\
\text { play sports? }\end{array}$ \\
\hline 3 & Coughing & Do you cough because of your asthma? \\
\hline 4 & Waking up at night & Do you wake up during the night because of your asthma? \\
\hline $\begin{array}{l}\text { Question answered by caregiver (score range: 0-5; 0: Everyday, 1: 19-24 days/month, 2: 11-18 } \\
\text { days/month, 3: 4-10 days/month, 4: 1-3 days/month, 5: Not at all) }\end{array}$ \\
\hline 5 & Daytime asthma symptoms & $\begin{array}{l}\text { During the last 4 weeks, on average, how many days per month did } \\
\text { your child have any daytime asthma symptoms? }\end{array}$ \\
\hline 6 & Wheezing & $\begin{array}{l}\text { During the last 4 weeks, how many days per month did your child } \\
\text { wheeze during the day because of asthma? }\end{array}$ \\
\hline 7 & Waking up at night & $\begin{array}{l}\text { During the last 4 weeks, on average, how many days per month did } \\
\text { your child wake up during the night because of asthma? }\end{array}$ \\
\hline
\end{tabular}

\section{Criteria used to ascertain asthma exacerbation}

135 (1) Symptoms of wheezing, aggravated coughing, feeling breathless, fast breathing, difficulties in sleeping reported by the participant and/or their caregiver(s);

Lung auscultation revealed wheezing,

138 (3) PEF value recorded in the asthma diary showed a significant decrease compared to stable 139 periods; or

140 (4) Measurements of spirometry or impulse oscillometry demonstrated a clinically meaningful 141 decrease. 


\section{Statistical analysis}

Linear mixed-effect regression (LMER) models were constructed to determine the exposureresponse association of personal air pollutant exposures with pulmonary health outcomes following Formula 4. From the model output, we calculated percent change (and 95\% confidence interval) of the biomarkers associated with an IQR increase in personal pollutant exposures.

\section{Formula 4}

$$
\begin{aligned}
Y_{i j} \sim \beta_{0}+\beta_{1} \text { Pol }_{i j}+\beta_{2} \text { RH }_{i j}+\beta_{3} \text { Temp }_{i j}+\beta_{4} \text { Sex }_{i}+\beta_{5} \text { Age }_{i}+\beta_{6} \text { EOS }_{i}+\beta_{7} \text { RI }_{i j}+\beta_{8} \text { ICS }_{i j} \\
+\beta_{9} \text { Sleep }_{i j}+\beta_{10} \text { Travel }_{i j}+\beta_{11} \text { Flare }_{i j}+\beta_{12} \text { Dust mite }_{i}+\beta_{13} \text { Ozone }_{i j}+W_{i} \\
+ \\
+P_{i}+\varepsilon
\end{aligned}
$$

\section{Codebook}

$i$ : Participant id number. $(i=1,2, \ldots, 43)$

$j$ : Sample number $(j=1,2,3,4)$

$Y_{i j}$ : Concentration of respiratory health outcome measures, including airway mechanics $\left(\mathrm{Z}_{5}, \mathrm{R}_{5}\right.$, $\mathrm{R}_{20}, \mathrm{R}_{5}-\mathrm{R}_{20}, \mathrm{X}_{5}$, Fres), lung function ( $\mathrm{FEV}_{1}, \mathrm{FVC}, \mathrm{PEF}, \mathrm{FEF}_{25-75}, \mathrm{FEV}_{1} / \mathrm{FVC}$ ), airway inflammation (FeNO), and asthma symptom scores (C-ACT scores). Note: FEV 1 , FVC, FEF $25-75$, $\mathrm{FEV}_{1} / \mathrm{FVC}$, FeNO were natural logarithm transformed.

Pol $_{i j}$ : 24-hour average personal exposure to $\mathrm{PM}_{2.5}$ mass and $\mathrm{PM}_{2.5}$ components $\left(\mu \mathrm{g} / \mathrm{m}^{3}\right)$ measured zero to three days prior to the clinical visit and the oxidative potential $(\mu \mathrm{g}$ Zymosan $/ \mathrm{m}^{3}$ ) of 24-hour average personal $\mathrm{PM}_{2.5}$ exposure measured during the same period (natural logarithm transformed).

Ozone $_{i j}$ : 24-hour average personal $\mathrm{O}_{3}$ exposure measured during the same time as $\mathrm{PM}_{2.5}$ (mass, components, or oxidative potential) exposures. (unit: ppb)

$R H_{i j}$ : 24-hour average ambient relative humidity measured zero to three days prior to health indicators measurement. (unit: \%)

$T e m p_{i j}$ : 24-hour average ambient temperature measured zero to three days prior to health indicator measurement, a natural spline with 3 degrees of freedom was applied. (unit: ${ }^{\circ} \mathrm{C}$ ) $\mathrm{Age}_{i}$ : Subject's age. (unit: year)

Sex $_{i}$ : Subject's sex. $(0=$ male, $1=$ female $)$

$\operatorname{EOS}_{i}$ : Baseline eosinophil number. (unit: $/ \mu \mathrm{L}$ )

$R I_{i j}$ : Upper respiratory tract infection like symptoms status during the two weeks prior to the clinical visit. ( $0=$ without respiratory infection symptoms, $1=$ with respiratory infection symptoms)

$I C S_{i j}$ : Inhaled corticosteroid usage status during the two weeks prior to the clinical visit. ( $0=$ not used, $1=$ used)

Sleep $p_{i j}$ : Sleep duration for the night prior to the clinical visit. (unit: hour)

Travel $_{i j}$ : Status of traveling to outside Shanghai City during the two weeks prior to each clinical visit. ( $0=$ no travel, $1=$ travel)

Flare $_{i j}$ : Status of asthma exacerbation during the two weeks prior to the clinical visit $(0=$ no exacerbation, $1=$ exacerbation)

Dust mite $_{i}$ : Dust mite allergy status $(0=$ not allergic, $1=$ allergic $)$

$W_{i}$ : Random intercept for the day of the week for clinical visit

$P_{i}$ : Individual-specific random intercept

$\varepsilon$ : Residual 


\section{5. Correlations among pollutant exposures}

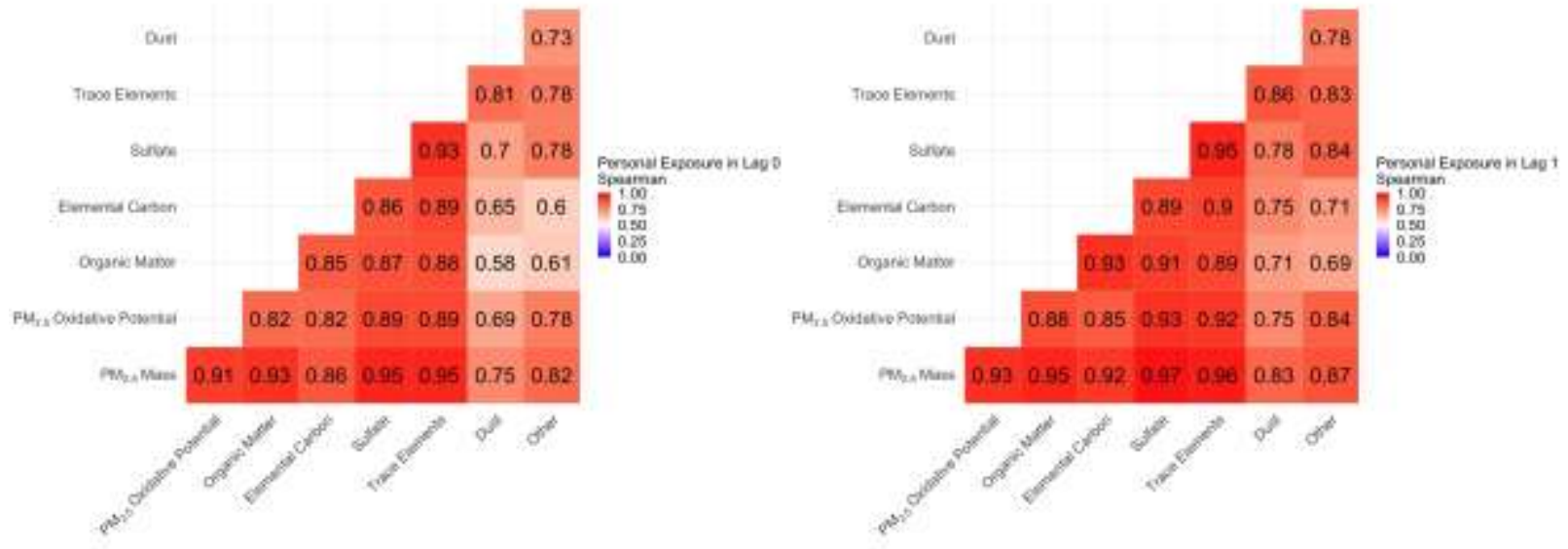

188

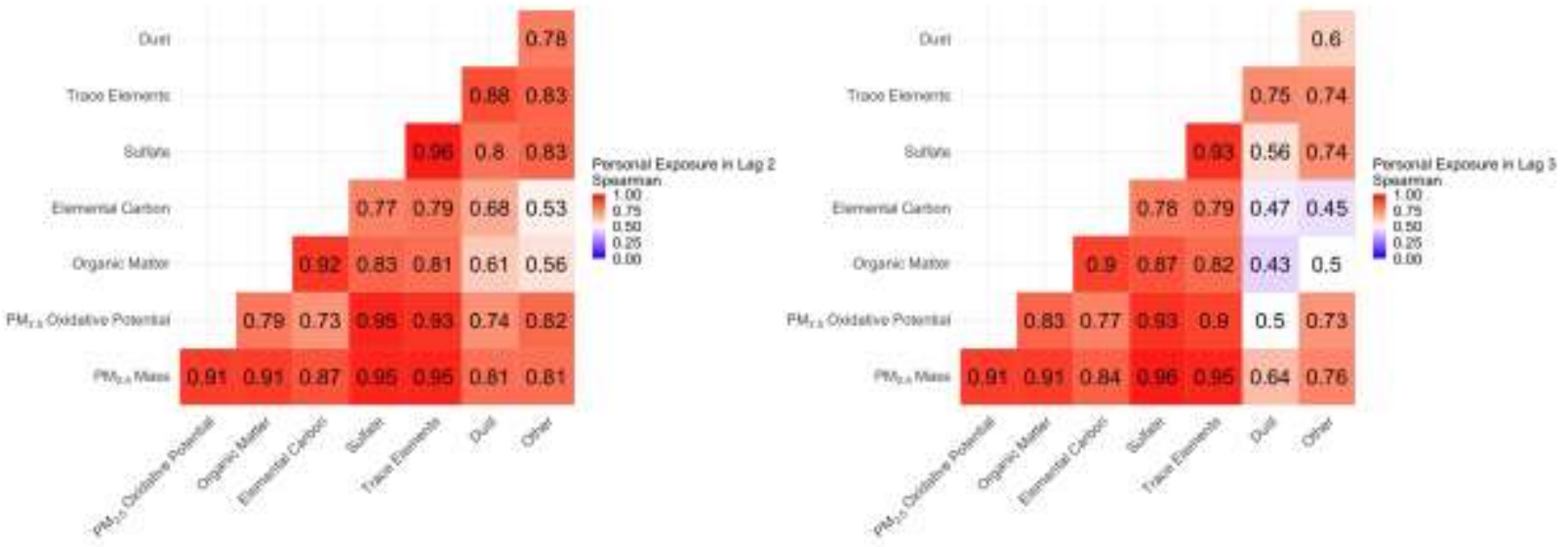

Figure S2. The correlations among air pollutant exposures. 

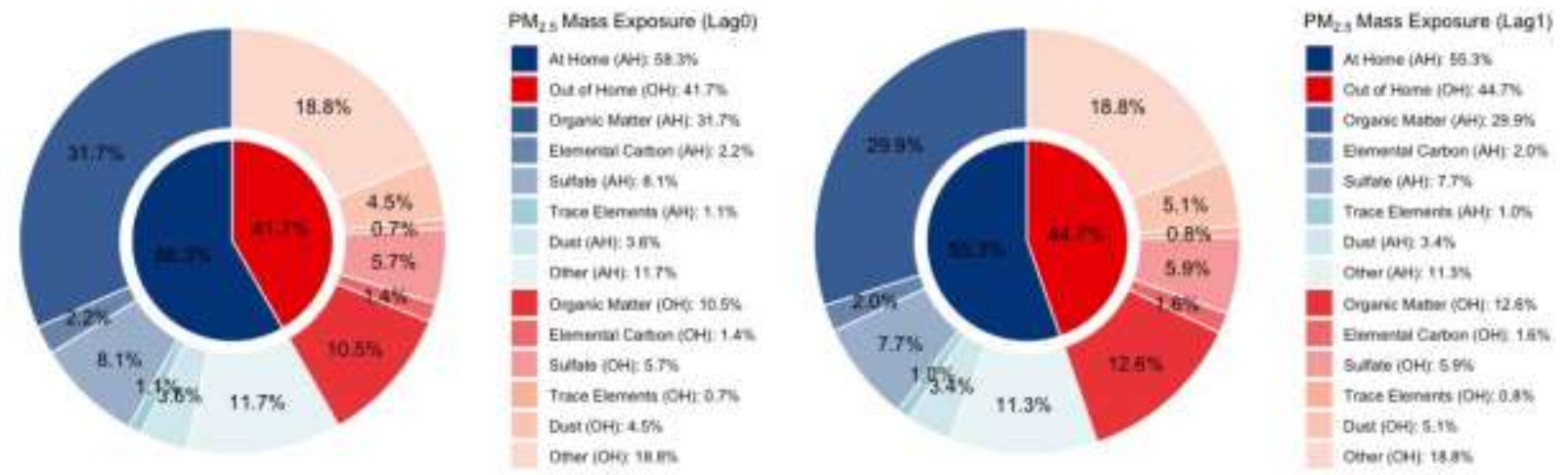

c
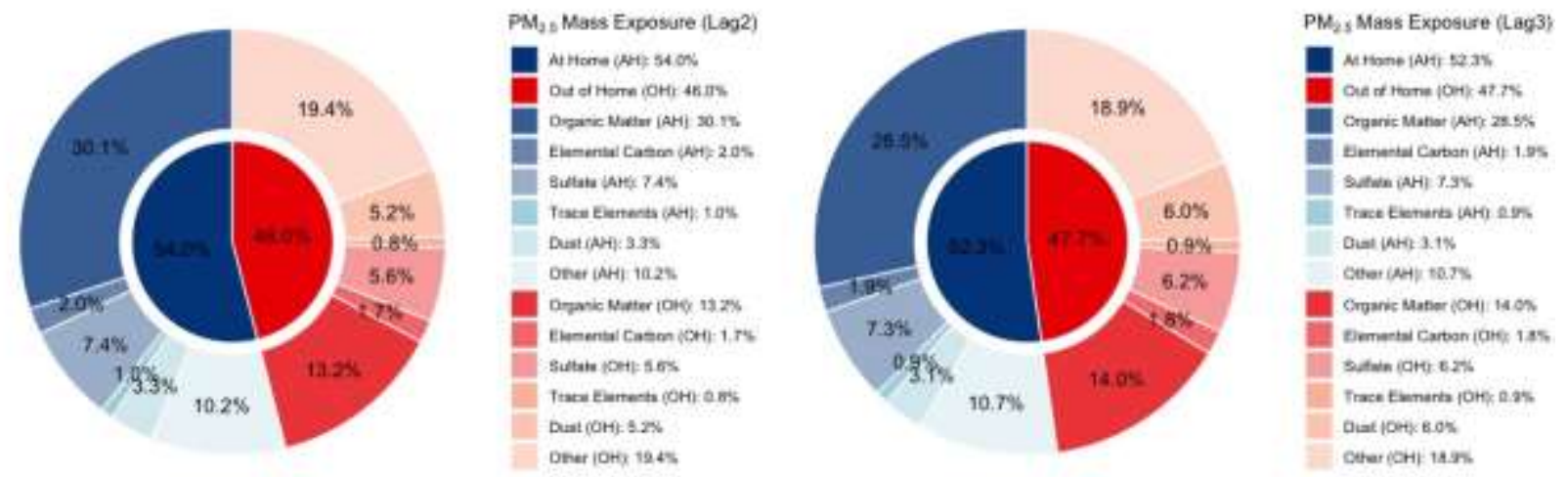

Figure S3. Median source contribution of personal exposure to $\mathrm{PM}_{2.5}$ mass and constituents from home and out of home. 
A

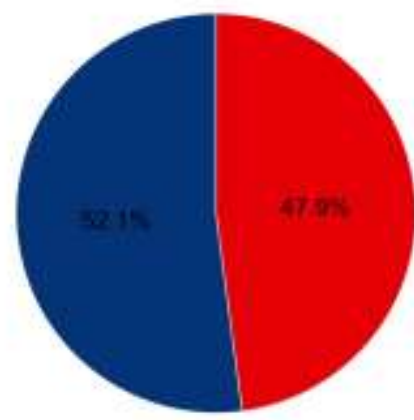

c

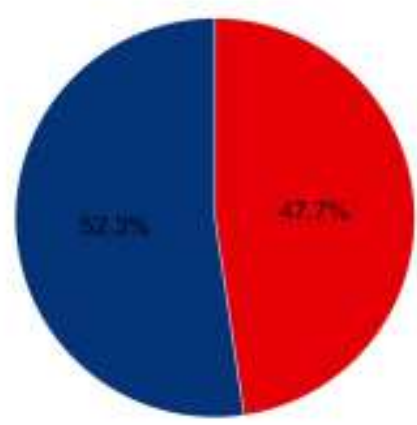

$P M_{23}$ Oxidative Potential \{Log 0 \}

At Horre (AH) $52.1 \%$
Out of Home $(\mathrm{OH}) .47 .9 \%$

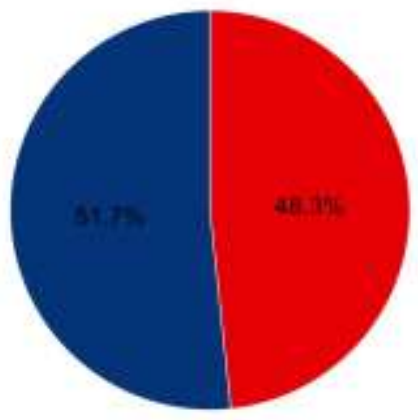

$\mathrm{PM}_{25}$ Oxidative Potential (Lag1)

Q At Home (AH): $51.7 \%$

Out of Home (OH) $48.3 \%$
$\mathrm{PMM}_{25}$ Oxidative Potential (L\&g2)

- At Home (AH) $52.3 \%$

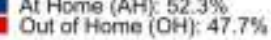

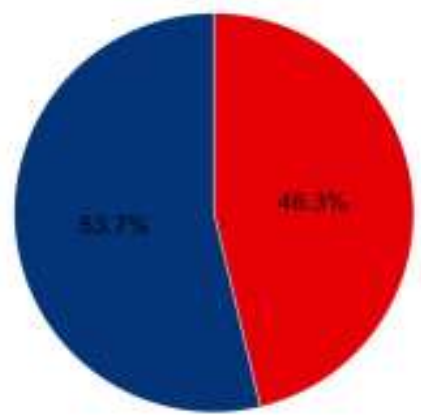

PM2, Oxidative Polential (Lag3]

At Home (AH): $53.7 \%$
Out of Home (OH): $46.3 \%$

Figure S4. Median source contribution of personal exposure to $\mathrm{PM}_{2.5}$ oxidative potential from 200 home and out of home.

201 
7. Detailed model results

203 Table S3. The model results of Figure 2.

\begin{tabular}{|c|c|c|c|c|c|c|c|c|c|}
\hline \multirow[t]{2}{*}{ Biomarker } & & \multicolumn{4}{|c|}{$\mathrm{PM}_{2.5}$ Mass } & \multicolumn{4}{|c|}{$\mathrm{PM}_{2.5}$ Oxidative Potential } \\
\hline & & Effect size & CI (lower) & CI (upper) & P value & Effect size & CI (lower) & CI (upper) & $P$ value \\
\hline $\mathrm{R}_{5}$ & $\operatorname{lag} 0$ & 4.4 & -1.1 & 9.9 & 0.115 & 4.8 & -1.2 & 10.7 & 0.117 \\
\hline $\mathrm{R}_{5}$ & $\operatorname{lag} 1$ & 2.6 & -3.0 & 8.2 & 0.357 & 2.9 & -2.8 & 8.6 & 0.310 \\
\hline $\mathrm{R}_{5}$ & $\operatorname{lag} 2$ & 7.2 & 0.1 & 14.3 & 0.047 & 9.0 & 2.5 & 15.4 & 0.007 \\
\hline $\mathrm{R}_{5}$ & $\operatorname{lag} 3$ & 5.9 & -1.0 & 12.7 & 0.093 & 7.1 & 0.5 & 13.6 & 0.036 \\
\hline $\mathrm{R}_{20}$ & $\operatorname{lag} 0$ & 3.0 & -1.8 & 7.9 & 0.221 & 4.5 & -0.8 & 9.7 & 0.094 \\
\hline $\mathrm{R}_{20}$ & lag 1 & 1.3 & -3.6 & 6.3 & 0.592 & 3.8 & -1.2 & 8.9 & 0.136 \\
\hline $\mathrm{R}_{20}$ & lag 2 & 3.9 & -2.4 & 10.1 & 0.223 & 4.7 & -1.0 & 10.5 & 0.104 \\
\hline $\mathrm{R}_{20}$ & $\operatorname{lag} 3$ & 4.8 & -1.1 & 10.7 & 0.110 & 5.8 & 0.2 & 11.5 & 0.044 \\
\hline $\mathrm{R}_{5}-\mathrm{R}_{20}$ & $\operatorname{lag} 0$ & 6.6 & -7.2 & 20.4 & 0.346 & 4.3 & -10.9 & 19.4 & 0.579 \\
\hline $\mathrm{R}_{5}-\mathrm{R}_{20}$ & $\operatorname{lag} 1$ & 5.8 & -8.5 & 20.1 & 0.425 & 2.1 & -12.4 & 16.6 & 0.777 \\
\hline $\mathrm{R}_{5}-\mathrm{R}_{20}$ & $\operatorname{lag} 2$ & 14.3 & -3.9 & 32.5 & 0.123 & 16.7 & 0.3 & 33.0 & 0.046 \\
\hline $\mathrm{R}_{5}-\mathrm{R}_{20}$ & $\operatorname{lag} 3$ & 8.0 & -9.4 & 25.4 & 0.366 & 9.8 & -7.0 & 26.7 & 0.251 \\
\hline $\mathrm{Z}_{5}$ & $\operatorname{lag} 0$ & 3.0 & -1.5 & 7.4 & 0.188 & 4.1 & -0.8 & 8.9 & 0.097 \\
\hline $\mathrm{Z}_{5}$ & $\operatorname{lag} 1$ & 2.1 & -2.4 & 6.6 & 0.365 & 3.1 & -1.5 & 7.7 & 0.185 \\
\hline $\mathrm{Z}_{5}$ & $\operatorname{lag} 2$ & 5.7 & 0.0 & 11.4 & 0.050 & 7.0 & 1.8 & 12.2 & 0.008 \\
\hline $\mathrm{Z}_{5}$ & lag 3 & 5.0 & -0.5 & 10.5 & 0.074 & 5.8 & 0.5 & 11.1 & 0.032 \\
\hline $\mathrm{X}_{5}$ & lag 0 & -2.7 & -28.1 & 22.7 & 0.837 & 0.2 & -27.5 & 28.0 & 0.986 \\
\hline $\mathrm{X}_{5}$ & lag 1 & 16.6 & -10.4 & 43.6 & 0.227 & 13.4 & -14.1 & 40.9 & 0.338 \\
\hline $\mathrm{X}_{5}$ & $\operatorname{lag} 2$ & 15.6 & -18.2 & 49.3 & 0.363 & 9.9 & -20.7 & 40.5 & 0.523 \\
\hline $\mathrm{X}_{5}$ & $\operatorname{lag} 3$ & -11.9 & -44.4 & 20.6 & 0.470 & -20.4 & -51.8 & 11.1 & 0.202 \\
\hline Fres & lag 0 & 0.6 & -6.9 & 8.1 & 0.876 & 1.5 & -6.7 & 9.7 & 0.712 \\
\hline Fres & $\operatorname{lag} 1$ & -4.1 & -11.9 & 3.7 & 0.303 & -2.9 & -10.8 & 5.0 & 0.473 \\
\hline Fres & $\operatorname{lag} 2$ & -6.5 & -16.2 & 3.3 & 0.190 & -3.5 & -12.3 & 5.4 & 0.442 \\
\hline Fres & $\operatorname{lag} 3$ & 5.6 & -4.0 & 15.2 & 0.254 & 7.3 & -2.0 & 16.7 & 0.123 \\
\hline
\end{tabular}

205 Table S4. The model results of Figure 3.

\begin{tabular}{|c|c|c|c|c|c|c|c|c|c|}
\hline \multirow[t]{2}{*}{ Biomarker } & & \multicolumn{4}{|c|}{$\mathrm{PM}_{2.5}$ Mass } & \multicolumn{4}{|c|}{$\mathrm{PM}_{2.5}$ Oxidative Potential } \\
\hline & & Effect size & CI (lower) & CI (upper) & $\mathrm{P}$ value & Effect size & CI (lower) & CI (upper) & P value \\
\hline $\mathrm{FEV}_{1}$ & $\operatorname{lag} 0$ & -0.6 & -2.7 & 1.5 & 0.556 & -0.6 & -2.8 & 1.7 & 0.621 \\
\hline $\mathrm{FEV}_{1}$ & $\operatorname{lag} 1$ & -2.0 & -4.1 & 0.1 & 0.066 & -2.4 & -4.6 & -0.2 & 0.030 \\
\hline $\mathrm{FEV}_{1}$ & $\operatorname{lag} 2$ & -0.2 & -2.9 & 2.6 & 0.900 & -0.3 & -2.8 & 2.3 & 0.835 \\
\hline $\mathrm{FEV}_{1}$ & $\operatorname{lag} 3$ & 0.1 & -2.4 & 2.7 & 0.916 & -0.3 & -2.7 & 2.1 & 0.786 \\
\hline FVC & $\operatorname{lag} 0$ & -1.0 & -2.6 & 0.7 & 0.238 & -0.7 & -2.5 & 1.0 & 0.414 \\
\hline FVC & $\operatorname{lag} 1$ & -1.2 & -2.8 & 0.5 & 0.156 & -1.7 & -3.4 & -0.1 & 0.041 \\
\hline FVC & $\operatorname{lag} 2$ & 0.0 & -2.0 & 2.0 & 0.977 & -0.1 & -2.0 & 1.8 & 0.907 \\
\hline FVC & $\operatorname{lag} 3$ & -1.0 & -2.9 & 1.0 & 0.328 & -1.1 & -2.9 & 0.8 & 0.267 \\
\hline $\mathrm{FEV}_{1} / \mathrm{FVC}$ & $\operatorname{lag} 0$ & 0.5 & -0.9 & 1.9 & 0.495 & 0.2 & -1.3 & 1.7 & 0.796 \\
\hline $\mathrm{FEV}_{1} / \mathrm{FVC}$ & $\operatorname{lag} 1$ & -0.7 & -2.0 & 0.7 & 0.346 & -0.7 & -2.1 & 0.8 & 0.368 \\
\hline $\mathrm{FEV}_{1} / \mathrm{FVC}$ & lag 2 & -0.1 & -1.9 & 1.8 & 0.940 & -0.3 & -2.0 & 1.4 & 0.688 \\
\hline $\mathrm{FEV}_{1} / \mathrm{FVC}$ & $\operatorname{lag} 3$ & 0.9 & -0.8 & 2.6 & 0.308 & 0.4 & -1.2 & 2.0 & 0.626 \\
\hline $\mathrm{FEF}_{25-75}$ & $\operatorname{lag} 0$ & -1.7 & -6.2 & 3.0 & 0.466 & -2.0 & -6.9 & 3.1 & 0.427 \\
\hline $\mathrm{FEF}_{25-75}$ & $\operatorname{lag} 1$ & -4.5 & -8.9 & 0.1 & 0.053 & -5.1 & -9.6 & -0.4 & 0.035 \\
\hline $\mathrm{FEF}_{25-75}$ & $\operatorname{lag} 2$ & -1.0 & -6.9 & 5.2 & 0.732 & -1.8 & -7.1 & 3.9 & 0.535 \\
\hline $\mathrm{FEF}_{25-75}$ & $\operatorname{lag} 3$ & 0.9 & -4.7 & 6.8 & 0.763 & -0.8 & -6.1 & 4.8 & 0.773 \\
\hline PEF & $\operatorname{lag} 0$ & 0.5 & -2.5 & 3.4 & 0.754 & 0.5 & -2.7 & 3.7 & 0.749 \\
\hline PEF & $\operatorname{lag} 1$ & -1.6 & -4.6 & 1.3 & 0.275 & -1.9 & -5.0 & 1.1 & 0.205 \\
\hline PEF & lag 2 & 0.4 & -3.4 & 4.1 & 0.851 & -0.4 & -3.8 & 3.1 & 0.831 \\
\hline PEF & $\operatorname{lag} 3$ & 1.5 & -2.0 & 5.1 & 0.391 & 0.1 & -3.3 & 3.5 & 0.956 \\
\hline FeNO & $\operatorname{lag} 0$ & 3.6 & -5.3 & 13.4 & 0.435 & 1.7 & -7.9 & 12.2 & 0.738 \\
\hline FeNO & $\operatorname{lag} 1$ & 3.7 & -5.3 & 13.5 & 0.430 & 3.8 & -5.4 & 13.8 & 0.432 \\
\hline FeNO & $\operatorname{lag} 2$ & 0.5 & -10.5 & 12.8 & 0.934 & 3.0 & -7.4 & 14.5 & 0.582 \\
\hline FeNO & $\operatorname{lag} 3$ & 8.4 & -2.7 & 20.7 & 0.140 & 7.6 & -3.1 & 19.4 & 0.167 \\
\hline
\end{tabular}


$207 \quad$ Table S5. The model results of Figure 4.

\begin{tabular}{|c|c|c|c|c|c|c|c|c|c|}
\hline \multirow[t]{2}{*}{ Biomarker } & & \multicolumn{4}{|c|}{$\mathrm{PM}_{2.5}$ Mass } & \multicolumn{4}{|c|}{$\mathrm{PM}_{2.5}$ Oxidative Potential } \\
\hline & & $\begin{array}{l}\text { Effect } \\
\text { size }\end{array}$ & $\begin{array}{l}\begin{array}{l}\text { CI } \\
\text { (lower) }\end{array} \\
\end{array}$ & $\begin{array}{l}\text { CI } \\
\text { (upper) }\end{array}$ & $\mathrm{P}$ value & $\begin{array}{l}\text { Effect } \\
\text { size }\end{array}$ & $\begin{array}{l}\text { CI } \\
\text { (lower) }\end{array}$ & $\begin{array}{l}\text { CI } \\
\text { (upper) }\end{array}$ & $\mathrm{P}$ value \\
\hline Asthma control & $\operatorname{lag} 0$ & -3.6 & -8.7 & 1.4 & 0.16 & -6.5 & -12.1 & -0.9 & 0.02 \\
\hline Asthma control & $\operatorname{lag} 1$ & -2.9 & -8.0 & 2.2 & 0.26 & -3.8 & -8.9 & 1.3 & 0.15 \\
\hline Asthma control & $\operatorname{lag} 2$ & -5.3 & -11.7 & 1.0 & 0.10 & -6.3 & -11.9 & -0.6 & 0.03 \\
\hline Asthma control & $\operatorname{lag} 3$ & -4.1 & -9.9 & 1.7 & 0.16 & -3.1 & -8.7 & 2.5 & 0.27 \\
\hline $\begin{array}{l}\text { Limitation of physical } \\
\text { activities }\end{array}$ & lag 0 & -2.3 & -9.1 & 4.4 & 0.49 & -2.8 & -10.4 & 4.7 & 0.46 \\
\hline $\begin{array}{l}\text { Limitation of physical } \\
\text { activities }\end{array}$ & $\operatorname{lag} 1$ & -5.9 & -12.7 & 0.9 & 0.09 & -6.4 & -13.2 & 0.5 & 0.07 \\
\hline $\begin{array}{l}\text { Limitation of physical } \\
\text { activities }\end{array}$ & $\operatorname{lag} 2$ & -2.9 & -12.0 & 6.1 & 0.52 & -3.0 & -11.1 & 5.1 & 0.47 \\
\hline $\begin{array}{l}\text { Limitation of physical } \\
\text { activities }\end{array}$ & $\operatorname{lag} 3$ & -3.5 & -11.5 & 4.4 & 0.38 & -4.7 & -12.3 & 2.9 & 0.23 \\
\hline Coughing & $\operatorname{lag} 0$ & -1.2 & -9.1 & 6.6 & 0.76 & -3.7 & -12.5 & 5.1 & 0.40 \\
\hline Coughing & $\operatorname{lag} 1$ & -7.5 & -15.7 & 0.7 & 0.07 & -7.0 & -15.3 & 1.2 & 0.09 \\
\hline Coughing & $\operatorname{lag} 2$ & -5.2 & -16.2 & 5.7 & 0.34 & -7.0 & -16.8 & 2.9 & 0.16 \\
\hline Coughing & $\operatorname{lag} 3$ & -2.8 & -12.1 & 6.6 & 0.56 & -7.2 & -16.1 & 1.7 & 0.11 \\
\hline Waking up at night & $\operatorname{lag} 0$ & -0.4 & -3.7 & 2.9 & 0.80 & -1.4 & -5.1 & 2.3 & 0.46 \\
\hline Waking up at night & $\operatorname{lag} 1$ & -1.8 & -5.1 & 1.4 & 0.27 & -2.2 & -5.5 & 1.1 & 0.19 \\
\hline Waking up at night & $\operatorname{lag} 2$ & -2.3 & -6.6 & 2.1 & 0.30 & -3.3 & -7.1 & 0.5 & 0.09 \\
\hline Waking up at night & $\operatorname{lag} 3$ & 0.4 & -3.4 & 4.2 & 0.84 & -1.1 & -4.7 & 2.6 & 0.56 \\
\hline $\begin{array}{l}\text { Total C-ACT score } \\
\text { (Child) }\end{array}$ & lag 0 & -1.5 & -5.4 & 2.4 & 0.44 & -3.4 & -7.8 & 1.0 & 0.12 \\
\hline $\begin{array}{l}\text { Total C-ACT score } \\
\text { (Child) }\end{array}$ & $\operatorname{lag} 1$ & -4.4 & -8.4 & -0.4 & 0.03 & -4.7 & -8.7 & -0.7 & 0.02 \\
\hline $\begin{array}{l}\text { Total C-ACT score } \\
\text { (Child) }\end{array}$ & $\operatorname{lag} 2$ & -3.8 & -9.1 & 1.5 & 0.16 & -4.6 & -9.4 & 0.2 & 0.06 \\
\hline $\begin{array}{l}\text { Total C-ACT score } \\
\text { (Child) }\end{array}$ & $\operatorname{lag} 3$ & -2.3 & -6.9 & 2.2 & 0.31 & -3.7 & -8.1 & 0.6 & 0.09 \\
\hline $\begin{array}{l}\text { Daytime asthma } \\
\text { symptoms }\end{array}$ & $\operatorname{lag} 0$ & -3.1 & -6.8 & 0.6 & 0.10 & -3.7 & -7.9 & 0.5 & 0.09 \\
\hline $\begin{array}{l}\text { Daytime asthma } \\
\text { symptoms }\end{array}$ & lag 1 & -1.2 & -4.9 & 2.6 & 0.54 & -1.7 & -5.5 & 2.1 & 0.38 \\
\hline $\begin{array}{l}\text { Daytime asthma } \\
\text { symptoms }\end{array}$ & lag 2 & -2.2 & -6.9 & 2.6 & 0.37 & -4.1 & -8.2 & 0.1 & 0.05 \\
\hline $\begin{array}{l}\text { Daytime asthma } \\
\text { symptoms }\end{array}$ & $\operatorname{lag} 3$ & -2.7 & -6.8 & 1.5 & 0.20 & -2.9 & -6.9 & 1.1 & 0.16 \\
\hline Wheezing & $\operatorname{lag} 0$ & -0.8 & -3.5 & 1.9 & 0.55 & -1.9 & -4.9 & 1.2 & 0.23 \\
\hline Wheezing & $\operatorname{lag} 1$ & -1.6 & -4.3 & 1.2 & 0.26 & -1.9 & -4.6 & 0.9 & 0.18 \\
\hline Wheezing & $\operatorname{lag} 2$ & -2.7 & -6.2 & 0.8 & 0.13 & -4.2 & -7.2 & -1.1 & 0.01 \\
\hline Wheezing & $\log 3$ & -1.6 & -4.7 & 1.4 & 0.28 & -2.2 & -5.1 & 0.6 & 0.13 \\
\hline Wakeing up at night & $\operatorname{lag} 0$ & -0.8 & -2.8 & 1.2 & 0.41 & 0.0 & -2.3 & 2.2 & 0.97 \\
\hline Wakeing up at night & $\operatorname{lag} 1$ & -0.6 & -2.7 & 1.5 & 0.56 & -0.1 & -2.2 & 2.0 & 0.92 \\
\hline Wakeing up at night & $\operatorname{lag} 2$ & 0.0 & -2.7 & 2.7 & 0.99 & 0.0 & -2.4 & 2.4 & 0.99 \\
\hline Wakeing up at night & $\operatorname{lag} 3$ & -0.3 & -2.7 & 2.1 & 0.81 & -0.1 & -2.4 & 2.2 & 0.93 \\
\hline $\begin{array}{l}\text { Total C-ACT score } \\
\text { (Caregiver) }\end{array}$ & lag 0 & -1.6 & -3.8 & 0.7 & 0.16 & -1.9 & -4.4 & 0.7 & 0.15 \\
\hline $\begin{array}{l}\text { Total C-ACT score } \\
\text { (Caregiver) }\end{array}$ & $\operatorname{lag} 1$ & -1.1 & -3.4 & 1.1 & 0.32 & -1.2 & -3.5 & 1.0 & 0.29 \\
\hline $\begin{array}{l}\text { Total C-ACT score } \\
\text { (Caregiver) }\end{array}$ & $\operatorname{lag} 2$ & -1.6 & -4.5 & 1.2 & 0.26 & -2.7 & -5.2 & -0.2 & 0.03 \\
\hline $\begin{array}{l}\text { Total C-ACT score } \\
\text { (Caregiver) }\end{array}$ & $\operatorname{lag} 3$ & -1.5 & -4.0 & 0.9 & 0.22 & -1.7 & -4.1 & 0.6 & 0.15 \\
\hline
\end{tabular}


8. Associations of health outcomes with $\mathrm{PM}_{2.5}$ composition exposures.

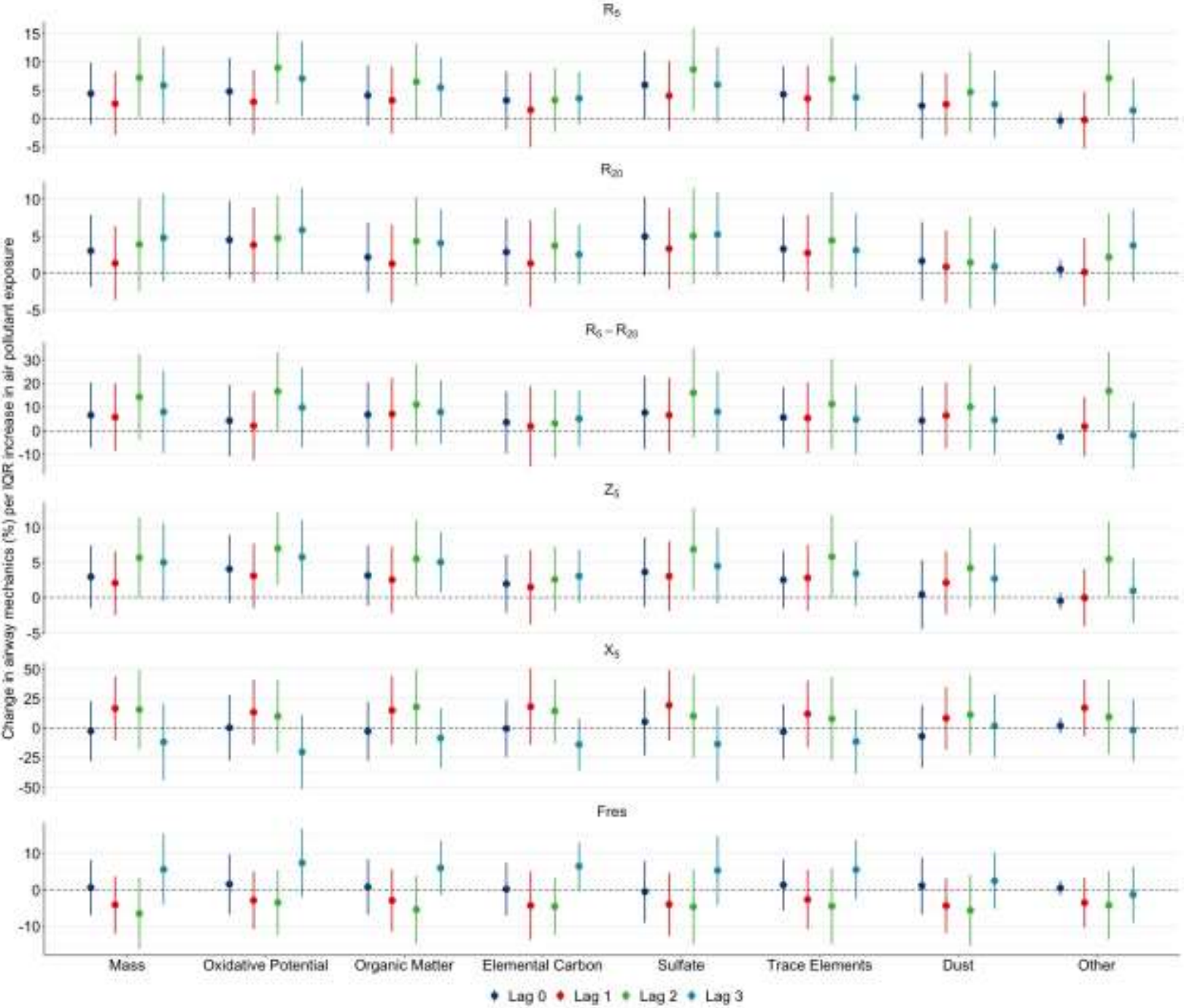

Figure S5. Estimated means and $95 \%$ confidence intervals for change in airway mechanics (\%) with one IQR increase in 24-hour average $\mathrm{PM}_{2.5}$ component exposure zero to three days prior to health outcome measurement (lag 0-3). 

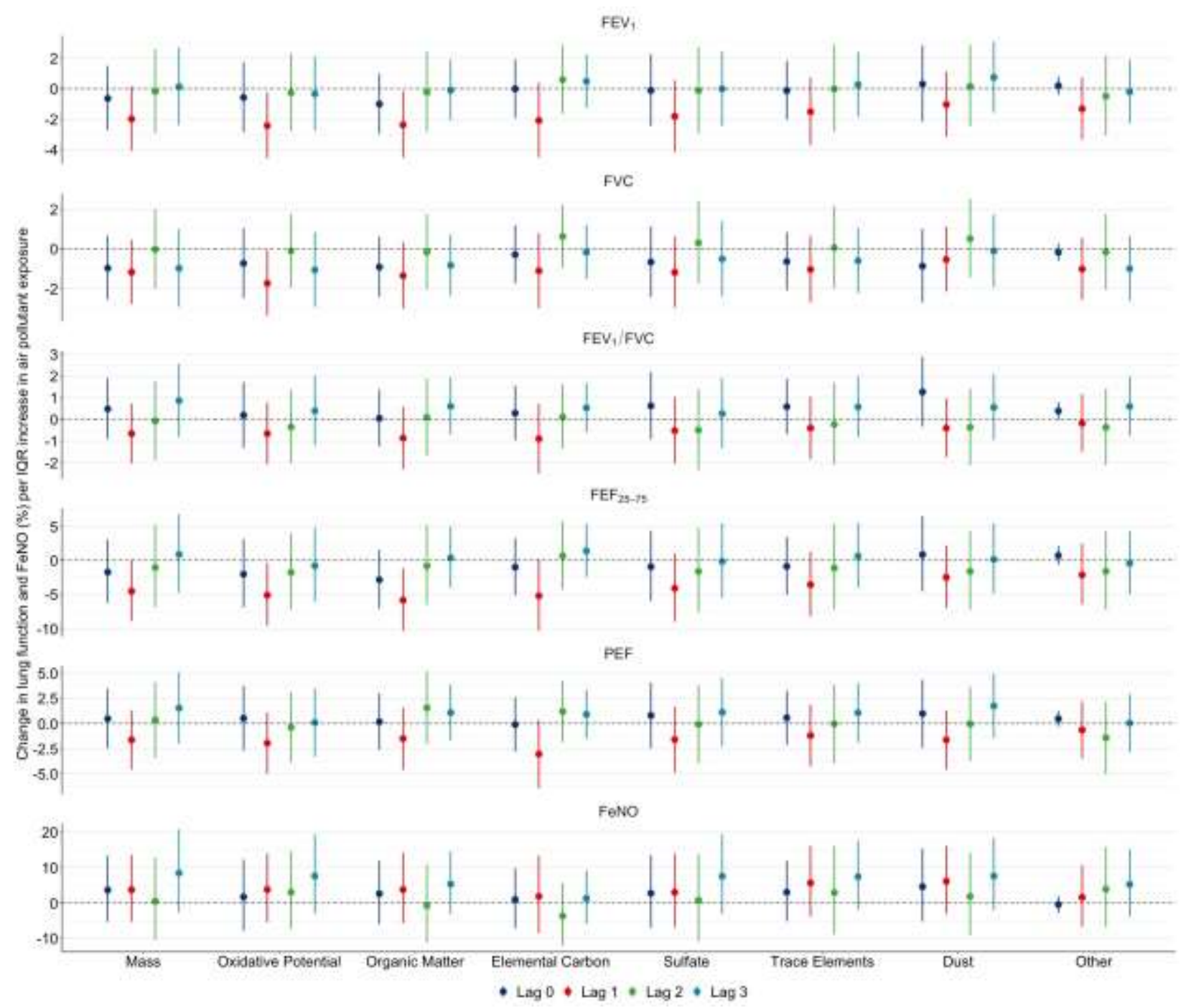

Figure S6. Estimated means and 95\% confidence intervals for change in lung function and

FeNO (\%) with one IQR increase in 24-hour average $\mathrm{PM}_{2.5}$ component exposure zero to three days prior to health outcome measurement (lag 0-3). 


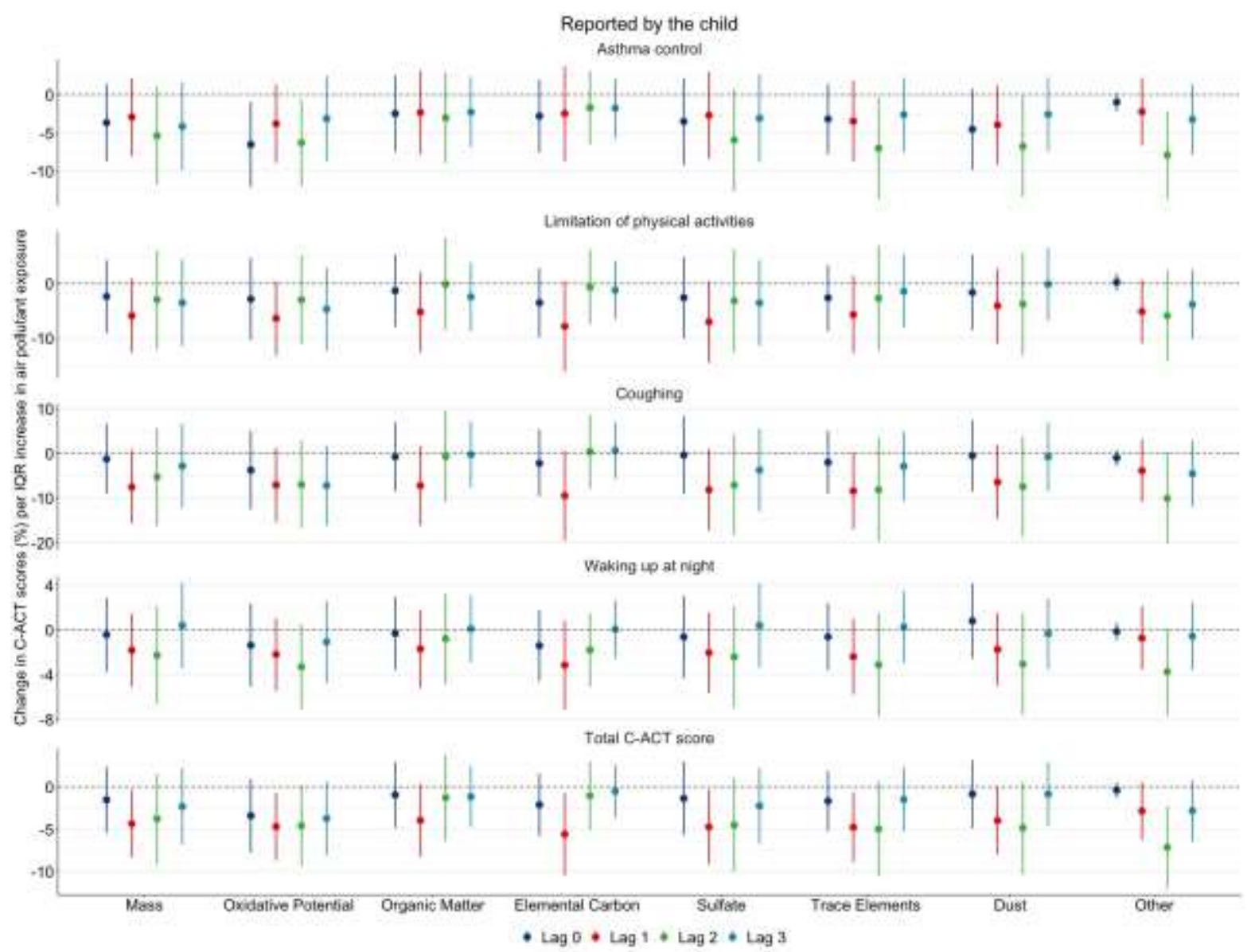

Figure S7. Estimated means and $95 \%$ confidence intervals for change in C-ACT scores reported 223 by child (\%) with one IQR increase in 24-hour average $\mathrm{PM}_{2.5}$ component exposure zero to three days prior to health outcome measurement (lag 0-3). 


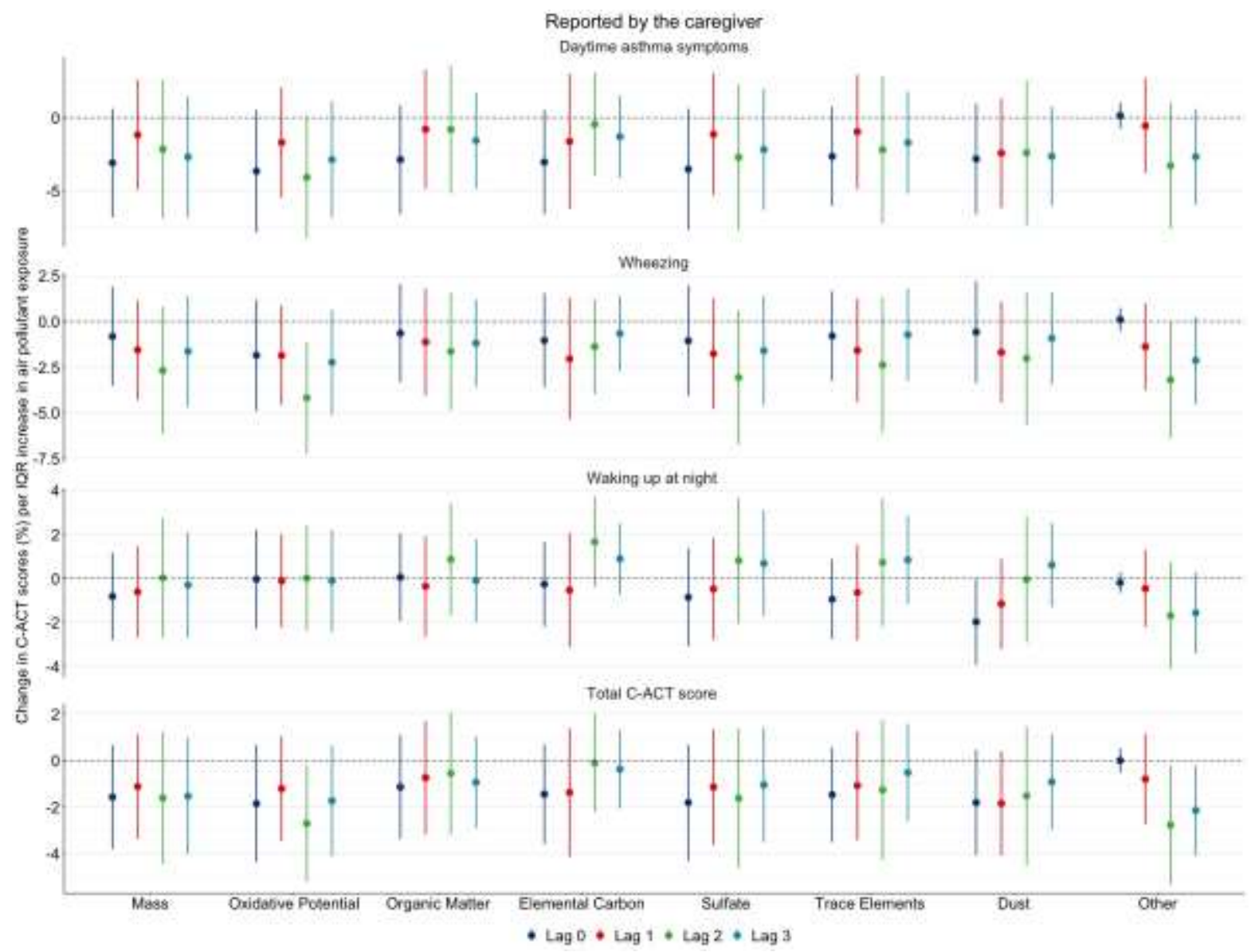

Figure S8. Estimated means and $95 \%$ confidence intervals for change in C-ACT scores reported 228 by caregiver (\%) with one IQR increase in 24-hour average $\mathrm{PM}_{2.5}$ component exposure zero to three days prior to health outcome measurement (lag 0-3). 


\section{Sensitivity analyses}

Sensitivity analyses were conducted for the associations shown in the main content in a 234 dataset excluding measurements of participants who had asthma exacerbation during the two weeks prior to the clinical visits following Formula 4. (Excluded 7 measurements from 6 participants)
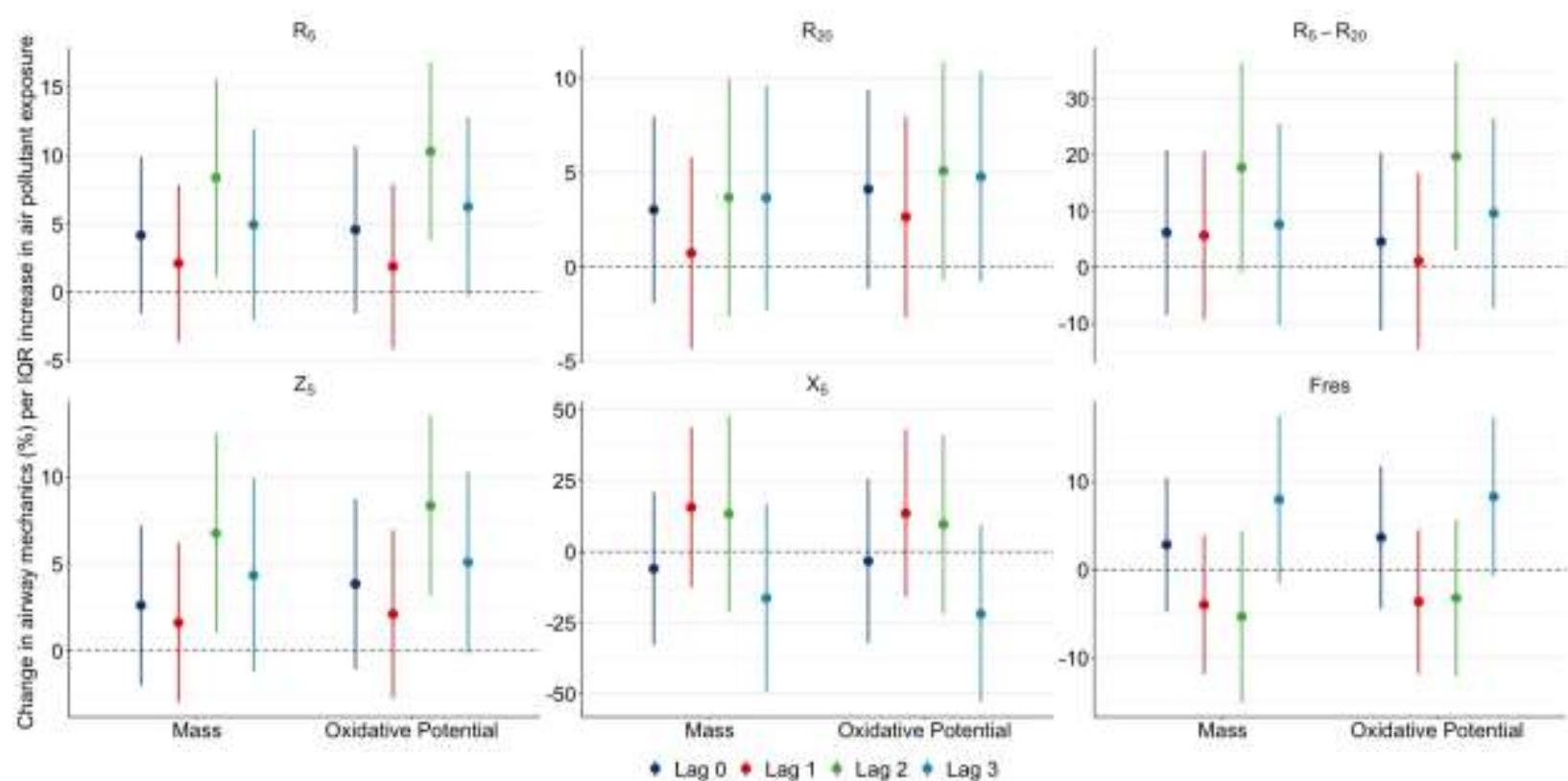

Figure S9. Estimated means and 95\% confidence intervals for change in airway mechanics (\%) with one IQR increase in 24-hour average $\mathrm{PM}_{2.5}$ mass exposure and $\mathrm{PM}_{2.5}$ oxidative potential zero to three days prior to health outcome measurement (lag 0-3). (Excluded asthma exacerbation) 

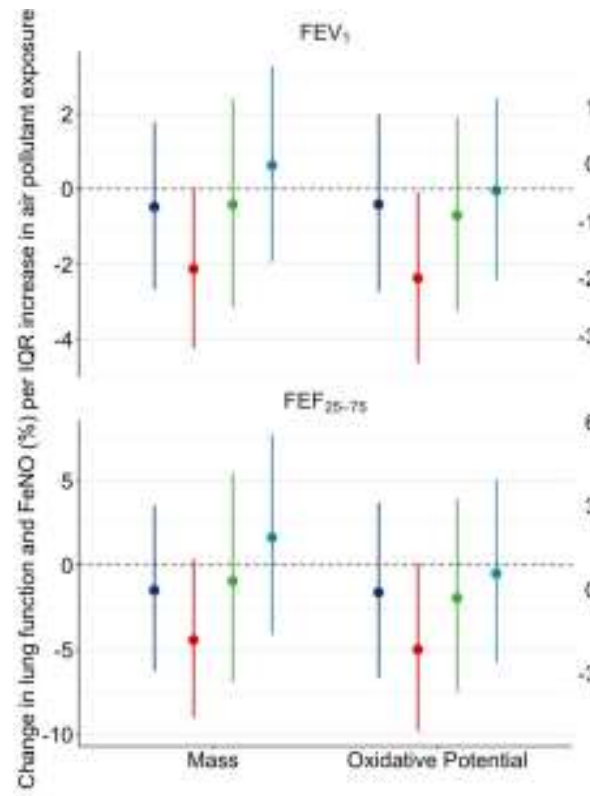

Figure S10. Estimated means and 95\% confidence intervals for change in lung function and airway inflammation (\%) with one IQR increase in 24-hour average $\mathrm{PM}_{2.5}$ mass exposure and $\mathrm{PM}_{2.5}$ oxidative potential zero to three days prior to health outcome measurement (lag 0-3). (Excluded asthma exacerbation)

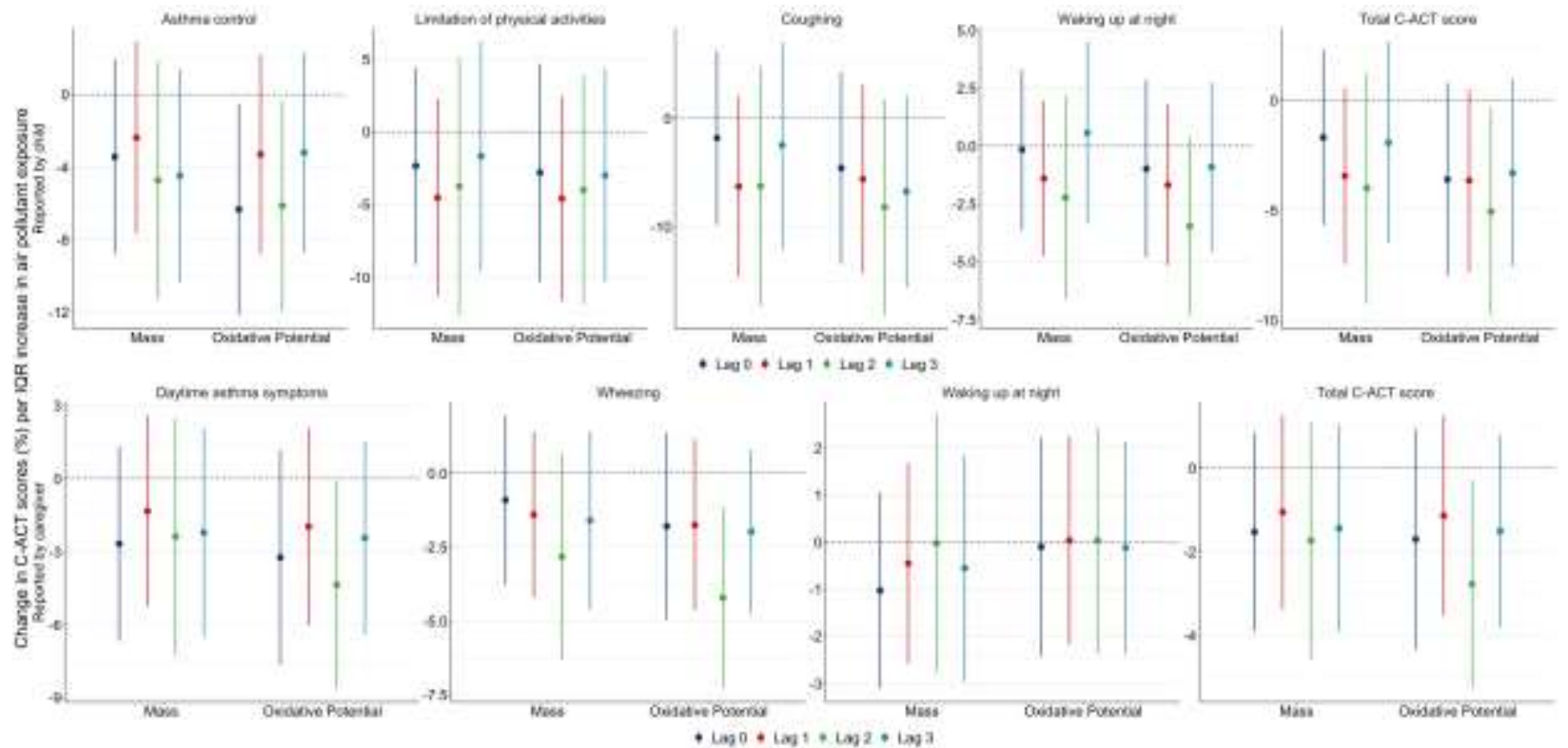

Figure S11. Estimated means and 95\% confidence intervals for change in C-ACT scores (\%) with one IQR increase in 24-hour average $\mathrm{PM}_{2.5}$ mass exposure and $\mathrm{PM}_{2.5}$ oxidative potential zero to three days prior to health outcome measurement (lag 0-3). (Excluded asthma exacerbation) 


\section{References:}

258 1. He, L.; Li, Z.; Teng, Y.; Cui, X.; Barkjohn, K. K.; Norris, C.; Fang, L.; Lin, L.; Wang, 259 Q.; Zhou, X.; Hong, J.; Li, F.; Zhang, Y.; Schauer, J. J.; Black, M.; Bergin, M.; Zhang, J.

260 Associations of personal exposure to air pollutants with airway mechanics in children with 261 asthma. Environment International. 2020, 138, 105647; DOI 10.1016/j.envint.2020.105647.

262 2. Brehmer, C.; Norris, C.; Barkjohn, K. K.; Bergin, M. H.; Zhang, J.; Cui, X.; Zhang, Y.; 263 Black, M.; Li, Z.; Shafer, M.; Schauer, J. J. The impact of household air cleaners on the chemical 264 composition and children's exposure to PM2. 5 metal sources in suburban Shanghai.

265 Environmental Pollution. 2019, 253, 190-198; DOI 10.1016/j.envpol.2019.07.003.

266 3. Brehmer, C.; Norris, C.; Barkjohn, K. K.; Bergin, M. H.; Zhang, J.; Cui, X.; Teng, Y.; 267 Zhang, Y.; Black, M.; Li, Z.; Shafer, M.; Schauer, J. J. The impact of household air cleaners on 268 the oxidative potential of PM2. 5 and the role of metals and sources associated with indoor and 269 outdoor exposure. Environmental Research. 2020, 181, 108919; DOI

270 10.1016/j.envres.2019.108919.

271 4. Liu, A. H.; Zeiger, R.; Sorkness, C.; Mahr, T.; Ostrom, N.; Burgess, S.; Rosenzweig, J. 272 C.; Manjunath, R. Development and cross-sectional validation of the Childhood Asthma Control 273 Test. Journal of Allergy and Clinical Immunology. 2007, 119 (4), 817-825; DOI

$274 \quad 10.1016 /$ j.jaci.2006.12.662. 